\title{
I 45 Cardiac magnetic resonance based assessment of cardiac work by pressure-volume and stress-length relation in patients with dilated cardiomyopathy and aortic valve stenosis Peter Alter*, Heinz Rupp, Marga B Rominger, Klaus J Klose and Bernhard Maisch
}

Address: Philipps University, Marburg, Germany

* Corresponding author

from I th Annual SCMR Scientific Sessions Los Angeles, CA, USA. I-3 February 2008

Published: 22 October 2008

Journal of Cardiovascular Magnetic Resonance 2008, I0(Suppl I):A46 doi:I0.1 I86/I532-429X-10-SI-A46

This abstract is available from: http://jcmr-online.com/content/I0/SI/A46

(c) 2008 Alter et al; licensee BioMed Central Ltd.

\section{Introduction}

Dilated cardiomyopathy (DCM) is characterized by left ventricular (LV) dilatation of unknown cause. It remains unresolved whether a reduced cardiac performance results primarily from dilatation or impaired cardiomyocyte contractility. In experimental animals, cardiac work is derived from pressure-volume area and analyzed further using stress-length relations. We hypothesized that not only pressure volume loops but also stress-length diagrams can be derived from cardiac volume and cardiac mass as assessed by CMR and invasively measured pressure.

\section{Methods}

Left ventricular (LV) volume and myocardial mass were assessed in 7 patients with aortic valve stenosis (AS), 8 with dilated cardiomyopathy (DCM), and 8 controls using prospectively ECG-gated CMR. LV pressure was measured invasively in real-time. Thus, pressure-volume loops could be derived. Stroke work was assessed as area within the pressure-volume loop. LV wall stress was calculated using a thick-wall sphere model. Stress-length loops were calculated to quantify stress-length based work. Taking the LV geometry into account, the normalization with regard to ventricular circumference resulted in myocardial work.

\section{Results}

Patients with AS exhibited an increased LV myocardial mass when compared with controls $(\mathrm{P}<0.05)$. LV wall stress was increased in DCM, but not in AS. Stroke work of AS was unchanged when compared with controls, whereas DCM exhibited a significant depression $(\mathrm{P}<$ $0.05)$. Myocardial work was significantly reduced in both AS and DCM when compared with controls $(P<0.05)$, also after normalization $(\mathrm{P}<0.001)$.

\section{Conclusion}

The present approach shows for the first time the feasibility to obtain pressure-volume land stress-length relations in patients based on $\mathrm{LV}$ volumetric analysis as derived from CMR and LV pressure as assessed invasively. This permits to evaluate a well established experimental method for clinical use. Myocardial work was reduced in patients with DCM and noteworthy also in AS, while stroke work was reduced in DCM only. It is suggested that deterioration of myocardial work is crucial for the prognosis. Accordingly, these basic physiological procedures should be included in the clinical assessment of heart function. 